\title{
Factores que afectan la transferencia de embriones de alpacas (Vicugna pacos) a llamas (Lama glama)
}

\author{
Factors that affect the transfer of embryos from alpacas (Vicugna pacos) to llamas \\ (Lama glama)
}

\author{
Uri Perez G. ${ }^{1,3}$, Eloy Gonzáles G. ${ }^{1}$, Rito Huayta A. ${ }^{2}$, María Apaza T. ${ }^{2}$, \\ Yesenia Quispe B. ${ }^{2}$, Manuel Pérez D. ${ }^{2}$
}

\section{RESUMEN}

El objetivo del estudio fue evaluar los factores que afectan la transferencia de embriones obtenidos a ovulación simple en alpacas y transferidos a alpacas y llamas. El estudio se realizó en condiciones del Altiplano peruano durante la época reproductiva (diciembre a marzo). Se seleccionaron 13 alpacas donadoras (Huacaya=6, Suri=7) y 73 receptoras (37 alpacas y 36 llamas) con al menos 15 días posparto. Las tasas de recuperación de embriones en los 93 lavados consecutivos fueron de $88.68 \%$ en alpacas Huacaya y de $80 \%$ en alpacas Suri, y las tasas de preñez fueron de 67.57 y $66.67 \%$ en alpacas y llamas, respectivamente sin diferencias significativas en ambos casos. Se evaluaron las variables tamaño y calidad del embrión, ubicación y área del del cuerpo lúteo (CL) en la receptora, especie de la receptora (llama, alpaca), raza de donadora (Huacaya, Suri), condición corporal (CC) de la receptora, profundidad del lugar de deposición del embrión (total, parcial), y cuerno uterino de deposición del embrión (derecho, izquierdo). La regresión logística indicó que los factores relacionados al mayor éxito de la preñez fueron la calidad del embrión (grado 1 y 2), la raza de la donadora (Huacaya) y la CC de la receptora (grado 2 y 3 ).

Palabras clave: cuerpo lúteo; transferencia de embriones; alpaca; llama

\section{AbSTRaCT}

The aim of this study was to evaluate the factors that affect the transfer of embryos obtained through simple ovulation in alpacas and transferred to alpacas and llamas. The study was conducted under conditions of the Peruvian Highlands during the reproductive

\footnotetext{
${ }^{1}$ Laboratorio de Reproducción Animal, Centro de Investigación IVITA El Mantaro, Universidad Nacional Mayor de San Marcos, Huancayo, Perú

${ }^{2}$ Laboratorio de Reproducción Animal, Universidad Nacional del Altiplano, Puno, Perú

${ }^{3}$ E-mail: harguer19@hotmail.com
}

Recibido: 18 de febrero de 2019

Aceptado para publicación: 28 de noviembre de 2019 
season (December to March). Thirteen donor alpacas (Huacaya $=6$, Suri $=7$ ) and 73 recipients (37 alpacas and 36 llamas) with at least 15 postpartum days were selected. The embryo recovery rate in 93 consecutive washes were $88.68 \%$ in Huacaya alpacas and $80 \%$ in Suri alpacas, and the pregnancy rates were 67.57 and $66.67 \%$ in alpacas and llamas, respectively without significant differences in both cases. The variables size and quality of the embryo, location and area of the corpus luteum (CL) in the recipient, species of the recipient (llama, alpaca), donor breed (Huacaya, Suri), body condition score (BCS) of the receptor, depth of the place of deposition of the embryo (total, partial), and uterine horn of deposition of the embryo (right, left) were evaluated. The logistic regression indicated that the factors related to better success of pregnancy were the quality of the embryo (grade 1 and 2), the breed of the donor (Huacaya) and BCS of the recipient (grade 2 and 3 ).

Key words: corpus luteum; embryo transfer; alpaca; llama

\section{INTRODUCCIÓN}

La transferencia de embriones (TE) en los camélidos sudamericanos (CSA) ofrece el potencial de aprovechar la capacidad reproductiva de los donantes de alto mérito genético en los rebaños domésticos y de facilitar la conservación y repoblación de especies de camélidos silvestres. La TE es una herramienta valiosa para identificar y eliminar defectos genéticos o defectos hereditarios porque implica el rápido incremento de genética conocida y sistematiza una base de datos de parentesco (Sumar, 2013). No obstante, la multiovulación en alpacas muestra una respuesta variable, siendo así que solo el $33.5 \%$ de hembras responden a los estímulos hormonales para el desarrollo de múltiples folículos, muchos de los cuales presentan ovocitos con desarrollo anormal y deficiente maduración nuclear y citoplasmática (Vaughan et al., 2013).

Existen varios factores que afectan la TE en vacunos y ovinos, tanto por parte de las donantes y las receptoras, como del embrión y el ambiente uterino (Spell et al., 2001; Bari et al., 2003; Hidalgo et al., 2004). Entre las técnicas evaluadas, se dispone de la técnica de lavado uterino en alpacas con ovulación simple, que no requiere un tratamiento previo de multiovulación; por lo tanto, de menor costo, lográndose hasta $70 \%$ de efectividad de colección (Huanca et al., 2012; Vaughan, 2012).

La TE interespecie podría ser una herramienta posible para conservar especies en peligro cuando no hay un número suficiente de receptoras de una especie dada (Comizzoli et al., 2000; Amstislavsky, 2006). La TE interespecie podría ser una opción para lograr mejores tasas de gestación al transferir embriones de alpaca en llamas receptoras; de esta manera utilizar la mayor habilidad materna de la llama y lograr tasas de preñez de 57-70\% (Sumar et al., 2012; Sumar, 2013); una alternativa que requiere ser evaluada a nivel de pequeños y medianos productores, de allí que el objetivo del presente estudio fue evaluar los factores que afectan la TE interespecie a ovulación simple en alpacas y llamas en el altiplano peruano durante la época reproductiva.

\section{Materiales y Métodos}

\section{Animales}

El estudio se llevó acabo en la unidad Antacalla de la Empresa de Propiedad Social (EPS) «Rural Alianza», ubicada en el distrito de Nuñoa, departamento de Puno, Perú, 
a una altitud de $4230 \mathrm{msnm}$. Se seleccionaron donadoras no gestantes y no lactantes de las razas Huacaya $(n=6)$ y Suri $(n=7)$, que de acuerdo con el productor presentaban buena fertilidad y eran libre de enfermedades, especialmente del tipo reproductivo. Las receptoras fueron 37 alpacas (17 Suri y 20 Huacaya) y 36 llamas (19 K'ara y 17 Cha'ku) con al menos un parto y mayor a 15 días posparto. Los machos fueron reproductores del grupo élite del centro pecuario, de 3-5 años y con fertilidad comprobada.

\section{Manejo de Donadoras y Receptoras}

Se sincronizó la onda folicular de las donadoras y receptoras aplicando $\mathrm{GnRH}$ (acetato de buserelina, $0.0082 \mathrm{mg}$ en alpacas y $0.0096 \mathrm{mg}$ en llamas) en el día 0 y 9 días después un análogo de prostaglandina $\mathrm{F}_{2 \alpha}$ (PGF $0.048 \mathrm{mg}$ ). En las donadoras se inició el empadre en el día 11 y a los 8 días posteriores se realizó el lavado uterino mediante la técnica no quirúrgica (explicada más adelante) y luego se aplicó un análogo de prostaglandina $\mathrm{F}_{2 \alpha}$ (PGF $0.048 \mathrm{mg}$ ) para asegurar la luteolisis y el desarrollo de la siguiente onda folicular. A los 2 días posteriores de la PGF se volvió a realizar el empadre y procedimiento de lavado uterino y luteolisis, actividad que se repitió durante toda la época reproductiva (diciembre a marzo). En las receptoras se aplicó un análogo de GnRH (acetato de buserelina, $0.0082 \mathrm{mg}$ en alpacas y $0.0096 \mathrm{mg}$ en llamas) un día después (día 12) del empadre realizado en las donadoras.

\section{Recuperación de Embriones}

La recuperación de los embriones (8 días pos-empadre) se inició con la sujeción del animal en posición decúbito esternal y la aplicación intramuscular de xilazina clorhidrato $(1.5 \mathrm{mg} / \mathrm{kg})$ y acepromazina maleato $(0.02-$ $0.05 \mathrm{mg} / \mathrm{kg}$ ) como sedantes. Se evacuaron las heces del recto con la mano enguantada, se hizo la higiene de la región perianal y se introdujo la sonda Foley (N. $\left.{ }^{\circ} 14\right)$ guiada por un estilete de acero inoxidable. Una vez colocado a nivel del cuerpo del útero se insufló aire al balón de la sonda $(6-7 \mathrm{ml})$ para asegurarla y se procedió el lavado con el medio EmFlush II ambos cuernos en 4 a 5 oportunidades (20-40 ml), dependiendo del tamaño del útero de la donadora. La cantidad de medio fue controlada vía transrectal mediante la palpación y masaje de los cuernos para recuperar el medio con los embriones dentro un filtro EmCon (Agtech $\AA$ ) que fue vaciado en placas petri para su la búsqueda y evaluación de embriones (Sumar, 2013; Espezúa et al., 2015; Pacheco et al., 2016).

\section{Evaluación de Embriones}

La evaluación de embriones se realizó en un estereoscopio (40X) con platina atemperada a $25^{\circ} \mathrm{C}$. Los embriones fueron mantenidos en $500 \mu \mathrm{l}$ de medio de mantenimiento (Holding, Bioniche ${ }^{\circledR}$ ) contenidas dentro de la placa petri (6 pocillos). La evaluación se realizó según lo recomendado por el manual de la International Embryo Transfer Society-IETS (Stringfellow y Givens, 2011), considerando las categorías 1, 2, 3 y 4 . En forma complementaria, se midió el diámetro de los embriones colocando una escala métrica en milímetros por debajo de la placa Petri que contenía los embriones.

\section{Transferencia de Embriones}

La transferencia de embriones se realizó a continuación de la colecta (transferencia de embriones en fresco). Los embriones fueron sometidos a seis lavados sucesivos en placas de 6 pocillos y con el medio de mantenimiento antes de ser colocados dentro de las pajillas de TE (Sumar, 2013). Todos los embriones, independientemente de su categoría (1, 2, 3 o 4) fueron transferidos. Las receptoras fueron evaluadas mediante ecografía (Chison D600 VET $®$, transductor de $7.5 \mathrm{MHz}$ ) para determinar las características del cuerpo lúteo (área, diámetro, ubicación).

La TE se realizó de manera similar a la de obtención de embriones, donde la punta del aplicador de embriones es guiada por vía 
transrectal a través del cérvix llegando hasta el cuerno uterino (identificando la profundidad de TE: total o parcial). El embrión fue colocado en el cuerno uterino ipsilateral al cuerpo lúteo (CL) (Bourke et al, 1995; Trasorras et al., 2010; Sumar, 2013).

\section{Análisis Estadístico}

Las variables del estudio incluyeron el tamaño del embrión (mm), calidad del embrión (grado 1,2,3,4), ubicación del CL en la receptora (codificado en todas las variables discontinuas para el análisis en derecho:0, izquierdo:1), especie de la receptora (alpaca:0, llama:1), raza de la donadora (Suri:0, Huacaya:1), condición corporal (CC) según la Australian Alpaca Association (2008), profundidad del lugar de la TE (total, parcial), área del CL $\left(\mathrm{cm}^{2}\right)$ y lugar de la TE (cuerno derecho, izquierdo). La variable independiente fue la preñez (sí, no).

En la regresión logística binaria se utilizó la metodología de máxima verosimilitud descrita en estudios de producción de embriones en alpacas y vacunos (Peixoto et al., 2007; Vaughan et al., 2013) y se empleó para estimar el efecto de las variables sobre la probabilidad de preñez, siendo esta considerada como un evento binomial ( $1=$ preñez, $0=$ no preñez). Los análisis estadísticos fueron procesados mediante el programa estadístico R 3.4.4 (R Core, 2018).

\section{Resultados y Discusión}

Los resultados del análisis de tendencia central, dispersión y rangos sobre variables cuantitativas en donantes alpacas y receptoras alpacas y llamas se presentan en el Cuadro 1.

La tasa de recuperación de embriones en los lavados uterinos consecutivos (53 lavados en las alpacas Huacaya y 40 en las alpacas Suri) fue de $88.7 \%$ para donadoras
Huacaya y de $80 \%$ para las Suri, porcentajes superiores reportados en otros estudios donde los embriones fueron recuperados los días 7-7.5 post-cópula (Carnero et al., 2011; Pacheco et al., 2016). En el presente estudio se hizo la colección de los embriones en el día 8 , cuando se inicia el elongamiento de los embriones, aspecto de importancia en el reconocimiento materno y la prevención de la luteólisis, además, en este día, los embriones del cuerno derecho se encuentran migrando al cuerno izquierdo (Picha et al., 2012).

Las tasas de preñez fueron de $67.6 \%$ $(25 / 37)$ en alpacas y de $66.7 \%(24 / 36)$ en llamas. Por otro lado, Vaughan et al. (2013) lograron $77.8 \%$ de preñez con embriones recuperados a los 8 días de la cópula y Carnero et al. (2011) obtuvieron 60 y $75 \%$ al realizar la TE ipsilateral, en tanto que Pacheco et al. (2016) reportan $37.8 \%$ al hacer la TE en interespecie utilizando llamas como receptoras.

La regresión logística analizó variables que podrían influir sobre la preñez o no preñez durante el proceso de transferencia de embriones (Cuadro 2), siendo significativo la raza de la donadora y la $\mathrm{CC}$ de la receptora.

\section{Factores que afectan la TE interespecie (Donadoras)}

El tamaño de embrión fue de $1.89 \pm$ $0.92 \mathrm{~mm}$ y no tuvo significancia estadística dentro del modelo de la regresión logística. Estos promedios fueron superiores a los valores de $1.29 \pm 0.76 \mathrm{~mm}$ para ovulaciones simples y de $1.41 \pm 0.75 \mathrm{~mm}$ para ovulaciones múltiples (MOET) reportados por Vaughan et al. (2013), posiblemente debido a que dichos autores realizaron las colecciones entre el día 6 al día 9. La calidad embrionaria no fue significativa ( $\mathrm{p}=0.079$ ); $\sin$ embargo, la tendencia observada coincide con diversos autores, quienes demuestran que la calidad embrionaria es un factor de gran importancia para la gestación de los embriones transferidos, especialmente de embriones con categoría 1 y 2 con relación a embriones en 
Cuadro 1. Resultados del análisis de medidas de tendencia central, dispersión y rangos sobre variables cuantitativas en donantes alpacas y receptoras alpacas y llamas

\begin{tabular}{lccc}
\hline & $\mathrm{n}$ & Promedio \pm D.E. & Rango \\
\hline Tamaño de embrión $(\mathrm{mm})$ & 73 & $1.89 \pm 0.92$ & $0.4-5$ \\
Condición corporal $(1-5)$ & 73 & $3.44 \pm 0.45$ & $2.5-4.5$ \\
${\text { Área de cuerpo lúteo }\left(\mathrm{cm}^{2}\right)}^{\text {Calidad de embrión }}{ }^{1}$ & 73 & $1.15 \pm 0.28$ & $0.44-1.76$ \\
\hline
\end{tabular}

${ }^{1} 1,2,3$ o 4 (Stringfellow y Givens, 2011)

Cuadro 2. Resultados del análisis de la regresión logística sobre variables relacionadas con las donantes, las receptoras y el embrión sobre la preñez de alpacas y llamas utilizando embriones de alpacas

\begin{tabular}{lcccc}
\hline Variable & $\beta$ & $\begin{array}{c}\text { Error } \\
\text { estándar }\end{array}$ & $\mathrm{X}^{2}$ & $\mathrm{P}$ \\
\hline Tamaño del embrión & -0.075 & 0.078 & -0.958 & 0.346 \\
Calidad del embrión & 0.300 & 0.165 & 1.817 & 0.079 \\
Ubicación del CL (receptora) & 0.011 & 0.136 & 0.077 & 0.939 \\
Receptora (llama o alpaca) & 0.058 & 0.125 & 0.467 & 0.644 \\
Raza de donadora & 0.311 & 0.120 & 2.596 & $0.014^{*}$ \\
Condición corporal (receptora) & 0.988 & 0.291 & 3.400 & $0.002^{* *}$ \\
Profundidad del lugar de la TE & -0.002 & 0.186 & -0.011 & 0.991 \\
Área del CL (receptora) & 0.407 & 0.223 & 1.827 & 0.078 \\
Lugar de TE (derecha o izquierda) & 0.190 & 0.177 & 1.072 & 0.292 \\
\hline
\end{tabular}

$\mathrm{CL}$ : cuerpo lúteo; TE; transferencia de embriones; $\beta$ : coeficiente de regresión; ${ }^{*}: \mathrm{p}<0.05 ;{ }^{* *}$ : $p<0.01$

categoría 3 y 4 (Spell et al., 2001; Peixoto et al., 2007). En el presente estudio, la mayor proporción de embriones colectados fue de categoría $2(65.1 \%, 41 / 63)$, seguido embriones de categoría 1 y $3(14.28 \%$. 9/63) y de categoría $4(6.35 \%, 4 / 63)$. Por otro lado, las alpacas Huacaya presentaron mejores resultados como donadoras de embriones que las alpacas Suri $(\mathrm{p}<0.01)$, debido posiblemente a que los animales Suri son más susceptibles a la altura y a cambios bruscos de temperatura-ambiente (Bustinza, 2001; Quispe, 2018).

\section{Factores que afectan la TE interespecie (Receptoras)}

El número de CL desarrollados tanto en el ovario derecho como en el izquierdo fue similar en las receptoras (alpacas y llamas). En otros estudios se observa una actividad folicular alterna entre ovarios en el $80-85 \%$ de las observaciones (Chaves et al., 2002; Miragaya et al., 2004; Hancco et al., 2015). La raza de la receptora no fue determinante para el porcentaje de preñez. 
La CC es un factor importante en la mayor tasa de preñez, obteniéndose una mejor respuesta en animales con CC entre $2 \mathrm{y}$ 3 . Estos resultados se pueden correlacionar con lo que ocurre en bovinos durante el periodo periparto (animales con $\mathrm{CC}$ menor a $\mathrm{CC} 2$ ) donde se afecta la producción hormonal que controla la maduración folicular y ovulación, generándose un deterioro de la viabilidad del ovocito y en la función del CL (menor producción de progesterona); además de alterar las funciones de las células inmunes que aumentan la susceptibilidad a problemas uterinos (Britt, 1992).

La profundidad y el lugar de la TE en alpacas y llamas no tuvo influencia sobre la tasa de preñez, observándose tasas de preñez similares al transferir en el cuerno izquierdo (42.4\%) y en el cuerno derecho (44.3\%), resultado similar al obtenido por Carnero et al. (2011), posiblemente debido a la migración embrionaria del cuerno derecho a izquierdo (cuando la ovulación ocurre en el ovario derecho) que se observa en los camélidos sudamericanos (Fernandez-Baca et al., 1970), de modo que el embrión se ubica en el lugar apropiado para enviar la señal de reconocimiento materno (Trasorras et al., 2010; Vaughan et al., 2013).

El área del CL fue de $1.15 \pm 0.28 \mathrm{~cm}^{2}$, sin presentar una relación significativa con la tasa de preñez de las receptoras. Como se sabe, la gestación en este tipo de animales puede sostenerse con niveles adecuados de progesterona $(>3 \mathrm{nml} / \mathrm{l})$, sin tener relación con el tamaño del CL (Barnes, 2000; Rizos et al., 2012; Abalos et al., 2018).

\section{Conclusiones}

- La mayor tasa de preñez se obtiene con embriones de categoría 1 y 2 en comparación con los de categoría 3 y 4 .

- Las donadoras Huacaya proporcionan mejor calidad de embriones que las Suri.
- Animales con CC 2 y 3 tienen mayor probabilidad de mantener la preñez que aquellos con CC 1.4 o 5 .

\section{Agradecimientos}

Los autores agradecen a la EPS «Rural Alianza» y específicamente a la Unidad de Producción Antacalla, así como al Laboratorio de Reproducción Animal de la Facultad de Medicina Veterinaria y Zootecnia de la Universidad Nacional del Altiplano por las facilidades prestada para el desarrollo del estudio.

\section{Literatura Citada}

1. Abalos MC, Acuña F, Cancino AK, Aller JF. 2018. Effect of GnRH analogue administration on day 7 after natural mating on formation accessory corpus luteum, progesterone concentration and conception rate in llamas (Lama glama). Anim Reprod Sci 190: 47-52. doi: 10.1016/j.anireprosci.2018.01.006

2. Amstislavsky SY. 2006. Interspecies embryo and nuclei transfer as an approach to endangered mammalian species conservation. Russ J Dev Biol 37: 1-8. doi: 10.1134/S1062360406010012

3. Australian Alpaca Association. 2008. Alpaca fact sheet\#4: Body condition score (BCS) of alpacas. [Internet]. Available in: https://alpacalibrary.com/index.php/ husbandry-for-beginners/body-conditionfor-beginners/alpaca-fact-sheet-4-bodycondition-score-bcs-of-alpacas

4. Bari F, Khalid M, Haresign W, Murray A, Merrell B. 2003. Factors affecting the survival of sheep embryos after transfer within a MOET program. Theriogenology 59: 1265-1275. doi: 10.1016/S0093-691X(02)01162-7

5. Barnes FL. 2000. The effects of the early uterine environment on the subsequent development of embryo and fetus. Theriogenology 53: 649-658. doi: 10.1016/S0093-691X(99)00264-2 
6. Bourke D, Kyle C, McEvoy T, Young P, Adam C. 1995. Recipient synchronisation and embryo transfer in South American Camelids. Therioge-nology 43: 1-171.

7. Britt JH. 1992. Impacts of early postpartum metabolism on follicular development and fertility. Am Assoc Bovine Pract 24: 39-43.

8. Bustinza V. 2001. La alpaca. Puno, Perú: Univ. Nacional del Altiplano. 123 p.

9. Carnero SS, Huanca LW, Cordero RA, Vásquez EM, Huanca MT. 2011. Transferencia embrionaria ipsilateral y contralateral a la posición del cuerpo lúteo y supervivencia embrionaria en llamas. Rev Inv Vet Perú 22: 114-120. doi: 10.15381/rivep.v22i2.278

10. Chaves MG, Aba M, Agüero A, Egey J, Berestin V, Rutter B. 2002. Ovarian follicular wave pattern and the effect of exogenous progesterone on follicular activity in non-mated llamas. Anim Reprod Sci 69: 37-46. doi: 10.1016/ S0378-4320(01)00173-7

11. Comizzoli P, Mermillod P, Mauget R. 2000. Reproductive biotechnologies for endangered mammalian species. Reprod Nutr Dev 40: 493-504. doi: 10.1051/ rnd:2000113

12. Espezúa O, Chipayo Y, Olazabal J. 2015. Anestesia total intravenosa de la combinación xilacina, ketamina y fentanilo para canulación del primer compartimento en alpacas. Rev Investig Altoandinas 17: 41-46.

13. Fernández-Baca S, Hansel W, Novoa C. 1970. Embryonic mortality in the alpaca. Biol Reprod 3: 243-251. doi: 10.1093/biolreprod/3.2.243

14. Hancco EG, Llacsa J, Quispe YM, Pérez MG, Luque N, Pérez U. 2015. Dinámica folicular ovárica en alpacas de la raza suri (Vicugna pacos). Spermova 5: 51-54. doi: 10.18548/aspe/0002.11

15. Hidalgo CO, Gómez E, Prieto L, Duque P, Goyache F, Fernández L, Fernández I, et al. 2004. Pregnancy rates and metabolic profiles in cattle treated with propylene glycol prior to embryo transfer. Theriogenology 62: 664676. doi: $10.1016 / \mathrm{j}$.theriogenology.2003.11.006

16. Huanca T, Gonzáles M, Mamani-Cato $R$, Cárdenas O, Sapana $R$, Naveros M. 2012. Twin reciprocal embryo transfer between alpacas and llamas. In: ICAR. Satellite Meeting on Camelid Reproduction. Canada.

17. Miragaya MH, Aba MA, Capdevielle EF, Ferrer MS, Chaves MG, Rutter B, Agüero AA. 2004. Follicular activity and hormonal secretory profile in vicuna (Vicugna vicugna). Theriogenology 61: 663-671. doi: 10.1016/S0093-691X(03)00238-3

18. Pacheco J, Vélez V, Pezo D. 2016. Evaluación de la eficiencia de la transferencia de embriones interespecie entre alpacas y llamas obtenidos por ovulación simple. Rev Inv Vet Perú 27: 64-69. doi: 10.15381/rivep.v27i1.11464

19. Peixoto MG, Bergmann JA, Suyama E, Carvalho MR, Penna VM. 2007. Logistic regression analysis of pregnancy rate following transfer of Bos indicus embryos into Bos indicus $x$ Bos taurus heifers. Theriogenology 67: 287-292. doi: 10.1016/j.theriogenology.2006.06.012

20. Picha Y, Tibary A, Memon M, Kasimanickam R, Sumar J. 2012. Chronology of early embryonic development and embryo uterine migration in alpacas. Theriogenology 79: 702-708. doi: 10.1016/j.theriogenology.2012.11.027

21. Quispe J. 2018. Caracterización fenotípica de alpacas Suri conservadas en las comunidades de Huacochani e Hichocollo del departamento de La Paz. Rev Cient Inv INFO-INIAF 1: 37-42.

22. $R$ Core Team. 2018. R: a language and environment for statistical computing. $\mathrm{R}$ Foundation for Statistical Computing, Vienna, Austria. [Internet]. Available online at https://www.R-project.org/.

23. Rizos D, Scully S, Kelly AK, Ealy AD, Moros R, Duffy P, Al Naib A, et al. 2012. Effects of human chorionic gonadotrophin administration on day 5 after oestrus on corpus luteum charac- 
teristics, circulating progesterone and conceptus elongation in cattle. Reprod Fert Develop 24: 472-481. doi: 10.1071/ RD11139

24. Spell AR, Beal WE, Corah LR, Lamb GC. 2001. Evaluating recipient and embryo factors that affect pregnancy rates of embryo transfer in beef cattle. Theriogenology 56: 287-297. doi: 10.1016/S0093-691X(01)00563-5

25. Stringfellow D, Givens D, International Embryo Transfer Society. 2011. Manual of the International Embryo Transfer Society: a procedural guide and general information for the use of embryo transfer technology emphasizing sanitary procedures. $4^{\text {th }} \mathrm{ed}$. Illinois, USA: International Embryo Transfer Society.

26. Sumar J, Arellano P, Montenegro V, Londoñe P, Picha Y, Rodriguez C, Sanchez D, Torres R. 2012. Reciprocal embryo transfer in alpacas and llamas. In: ICAR. Satellite Meeting on Camelid Reproduction. Vancouver, Canada.
27. Sumar JB. 2013. Embryo transfer in domestic South American camelids. Anim Reprod Sci 136: 170-177. doi: 10.1016/ j.anireprosci.2012.10.029

28. Summers PM.1986. Collection, storage and use of mammalian embryos. Int Zoo Yb 24: 131-138. doi: 10.1111/j.17481090.1985.tb02528.x

29. Trasorras V, Chaves MG, Neild D, Gambarotta M, Aba M, Agüero A. 2010. Embryo transfer technique: Factors affecting the viability of the corpus luteum in llamas. Anim Reprod Sci 121: 279-285. doi: 10.1016/j.anireprosci.2010.06.004

30. Vaughan J. 2012. Embryo transfer in alpacas. In: ICAR. Satellite Meeting on Camelid Reproduction. Canada.

31. Vaughan J, Mihm M, Wittek T. 2013. Factors influencing embryo transfer success in alpacas - A retrospective study. Anim Reprod Sci 136: 194-204. doi: 10.1016/j.anireprosci.2012.10.010 